\title{
Rapidly Growing Squamous Cell Carcinoma of the Tongue
}

Dominick Myers ${ }^{1}$, Emily Allen ${ }^{1}$, Amr Essa ${ }^{1}$, Maryam Gbadamosi-Akindele ${ }^{1}$

1. Internal Medicine, Creighton University School of Medicine, Omaha, USA

Corresponding author: Amr Essa, amressa@creighton.edu

\begin{abstract}
The tongue can be a guide for different normal findings/variants, pathological lesions, or signs for systemic diseases. And oral cancer can be among the differentials, which can be detected early by a thorough oral exam. The early detection of oral cancer is particularly important, as the stage of oral cancer at the time of the diagnosis is the most critical factor determining the five-year survival. We present a case of a high-risk patient for oral cancers presented with a rapidly growing tongue lesion over six months that was diagnosed as a squamous cell carcinoma of the tongue on the biopsy. The case highlights an alarming rapid growth nature of oral cancers and alerts the clinicians of the importance of the physical exam as a cost-effective and potentially life-saving measure against oral cancers. It also demonstrates a brief review of risk factors and high-risk features of oral cancers.
\end{abstract}

Categories: Internal Medicine, Oncology, Dentistry

Keywords: oral cancers, tongue diseases, squamous cell carcinoma

\section{Introduction}

Oral cancers represent $3 \%$ of total cancer incidence in the United States, of which squamous cell carcinoma (SCC) is the most common one representing over $90 \%$. While the prognosis of many cancers has improved with medical advancements in recent years, the prognosis for oral cancers has not significantly changed [1]. A thorough periodic oral exam, especially in high-risk individuals and with precancerous lesions, is very cost-effective and can be life-saving as the stage of oral cancer at the time of the diagnosis is the most critical factor determining the five-year survival [1].

\section{Case Presentation}

A 62-year-old male presented to the clinic with a $2 \times 5 \mathrm{~cm}$ lesion on the right lateral tongue that was described as erythroplakia with ulceration. The lesion first appeared approximately six months ago after he had bitten his tongue during a fall and had been growing since then. He had a 50-pack year smoking history and consumed vodka daily. A punch biopsy revealed a squamous cell carcinoma, and he had a computed tomography (CT) scan of the head and neck (Figure 1) along with a total-body positron emission tomography (PET)/CT scan (Figure 2) for staging which was CT4aN1. He was scheduled for surgery that was delayed because of unforeseen circumstances on the patient's side. During his pre-operative evaluation, three months later, the lesion had been grown and measured $3 \times 6 \mathrm{~cm}$ (Figure 3). He had a subtotal glossectomy with a tracheostomy, bilateral neck dissection, and a radial forearm free flap. His pathology revealed perineural invasion and extracapsular extension. He was started on cetuximab plus radiation therapy regimen.

Myers et al. This is an open access article distributed under the terms of the Creative Commons Attribution License CC-BY 4.0., which permits unrestricted use, distribution, and reproduction in any medium, provided the original author and source are credited.

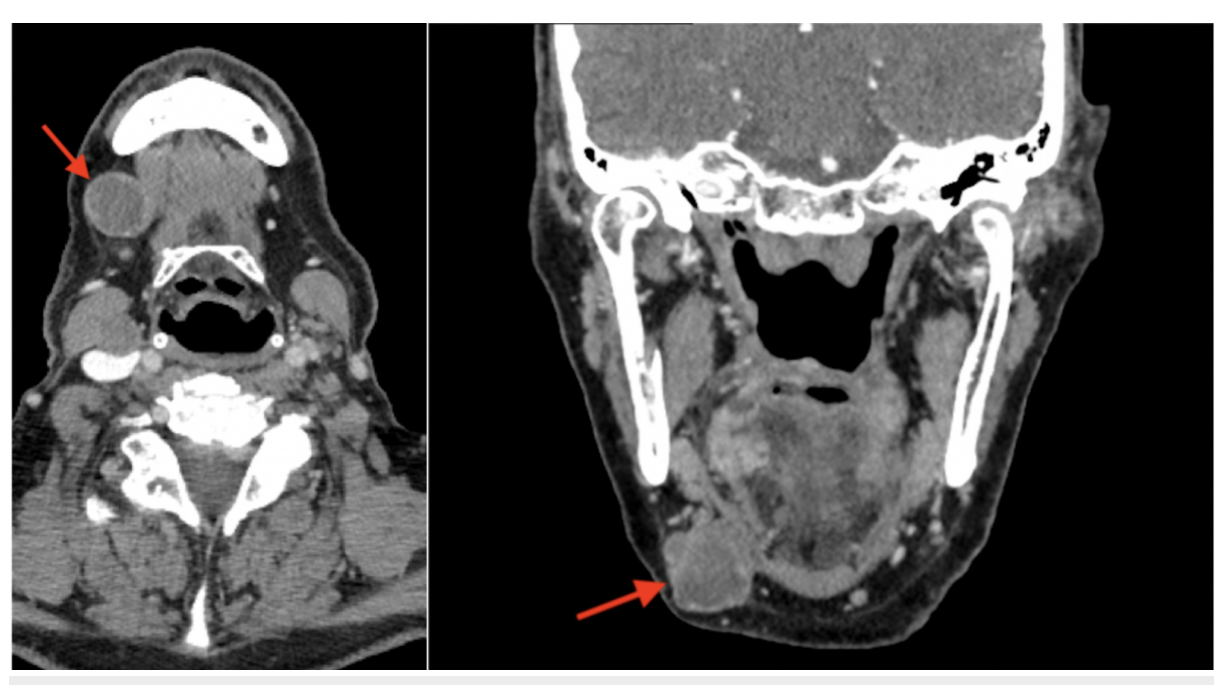




\section{Cureus}

FIGURE 1: Head and neck CT showing hypodense rim enhancing soft tissue lesion in the right submandibular space (red arrows).

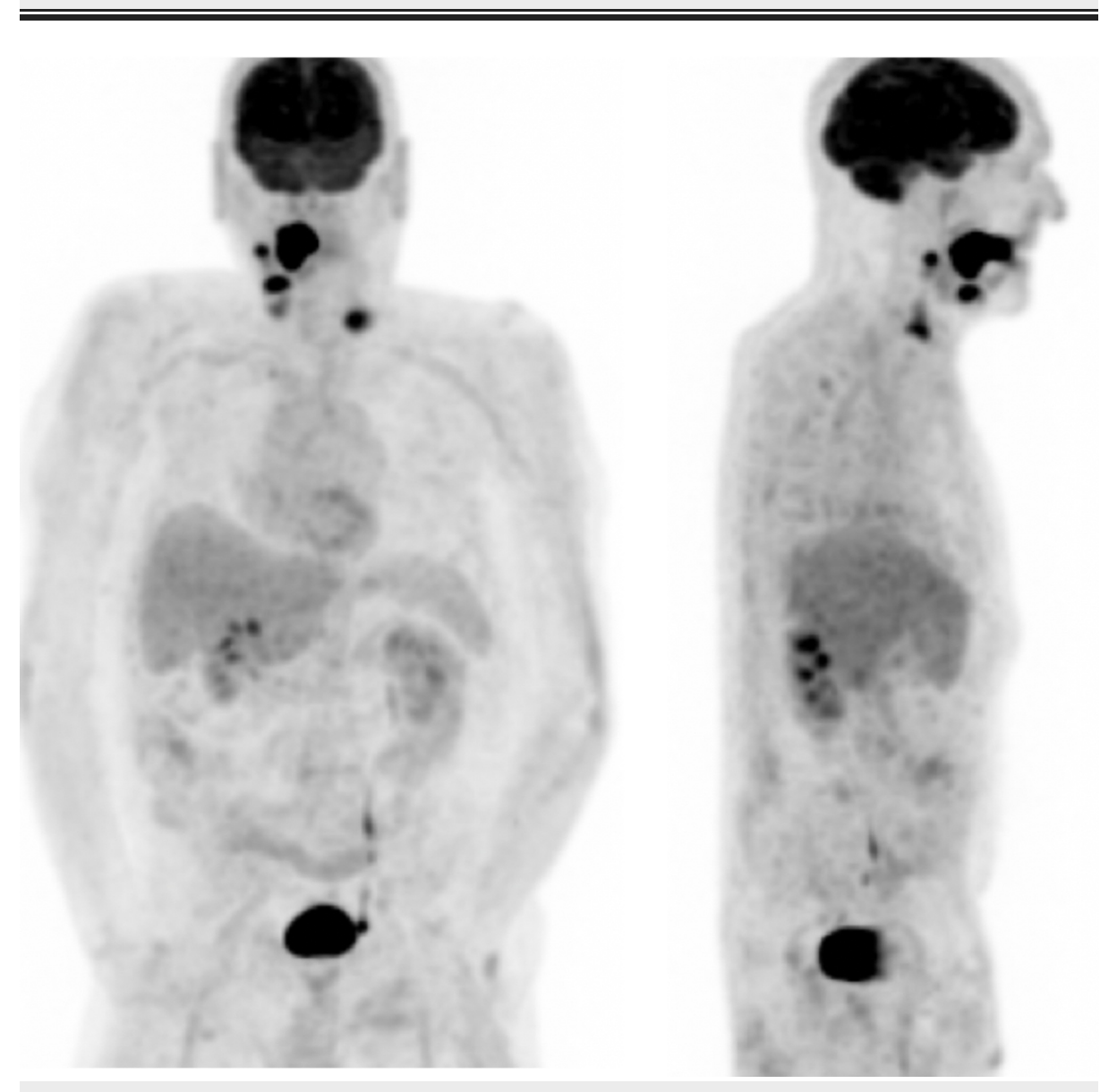

FIGURE 2: Whole body PET/CT scan showing increased metabolism in the tongue and nearby locations along with supraclavicular lymph node.

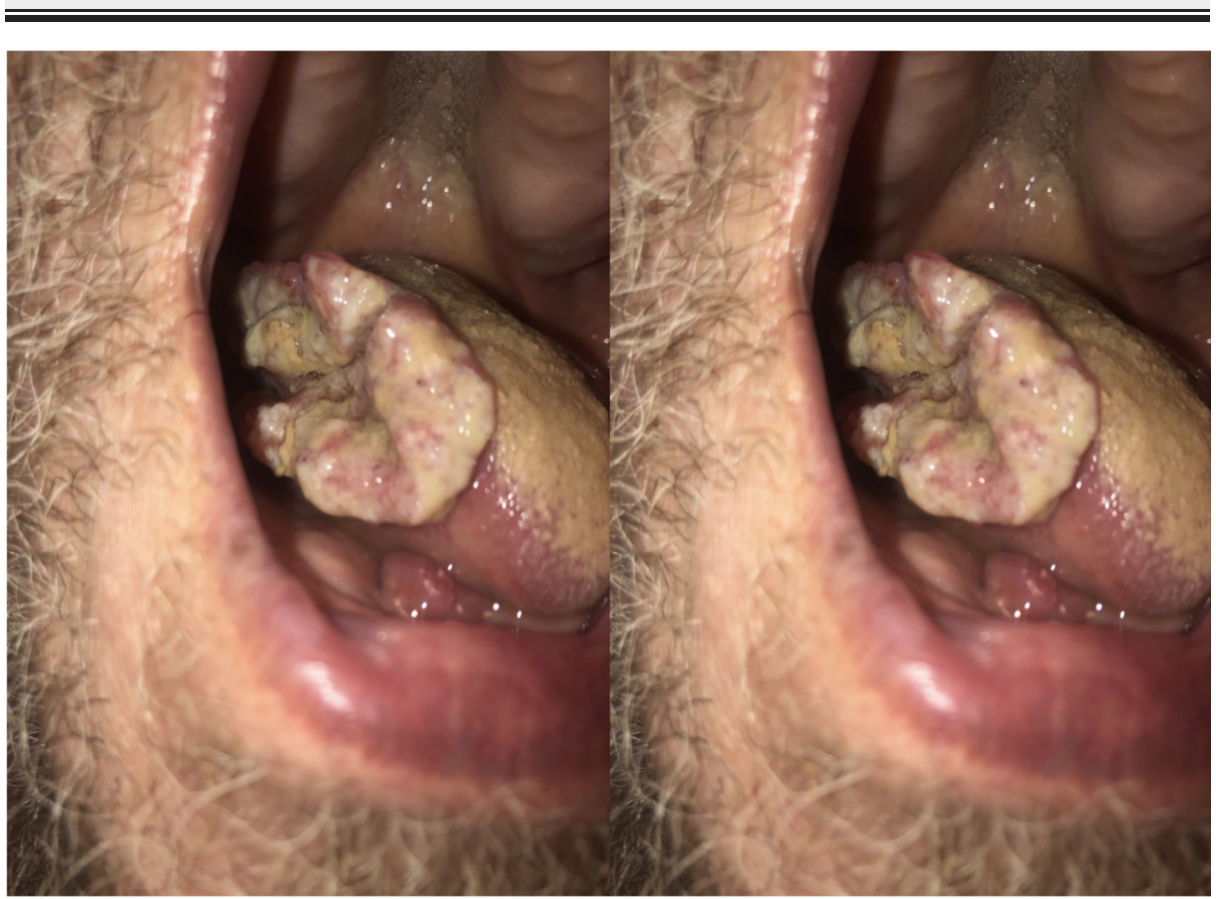




\section{Discussion}

Oral cancers can develop de novo, or from oral potentially malignant disorders (OPMDs) with leukoplakia and erythroplakia being the most common ones with a risk of malignant transformation of as high as $18 \%$ and $50 \%$, respectively [2]. Tobacco and smoking are the most common modifiable risk factors, and $80 \%$ of patients with oral cancers had a smoking history [1]. Also, drinking four or more alcohol drinks per day has a 9.29\% relative risk for developing oral cancer [3]. Further, the use of both of them concomitantly increases the risk by 100-fold in women and 38 in men [4]. Other risk factors have been identified, such as, immunosuppression, longstanding inflammation, lichen planus, human papilloma virus, and human immunodeficiency virus infection [5].

Our patient demonstrated a high-risk individual for developing oral cancer given his history of concomitant smoking and alcohol use, so a high index of suspicion should be warranted in evaluating such individuals. Also, his initial lesion of the lateral tongue might not have been readily visible on the oral exam; therefore in-depth oral exam is prompted as lesions can be obscured by oral structures and many oral cancer sites might not be visible on the initial exam, for example, the tongue posterior lateral and ventral surface which are the most common site for oral SCC [6]. So, during the evaluation of oral cavity for any lesions, any change in color, texture, or contour of the oral cavity should raise suspicion and a good way to remember the characteristics of high-risk lesions that might need a biopsy is any single mucosal lesion which is red and/or white, ulcer, lump or exceeding three weeks duration (RULE) [7].

Lastly, it is essential to understand the phenomenon of field cancerization in the development of oral cancer and precancerous lesions. Fields of altered cells can develop secondary to genetic mutations and long-term exposure of carcinogens. Those fields of cells can have normal morphology while the mucosa has acquired genetic alternations allowing malignant transformation. So, with more understanding of this phenomenon, it is vital to advise towards modifiable risk factor cessation, a thorough examination of the oral cavity and not only the morphologically abnormal lesion, and long-term follow-up as those fields can take few years to transform into cancer $[8,9]$.

\section{Conclusions}

Oral cancer lesions can be often asymptomatic until they are advanced, and the progression can occur rapidly. And regular, long-term screening for intraoral lesions in patients who are at high risk for oral cancer is a simple, cost-effective, and potentially life-saving preventative measure.

\section{Additional Information \\ Disclosures}

Human subjects: Consent was obtained by all participants in this study. Conflicts of interest: In compliance with the ICMJE uniform disclosure form, all authors declare the following: Payment/services info: All authors have declared that no financial support was received from any organization for the submitted work. Financial relationships: All authors have declared that they have no financial relationships at present or within the previous three years with any organizations that might have an interest in the submitted work. Other relationships: All authors have declared that there are no other relationships or activities that could appear to have influenced the submitted work.

\section{References}

1. Neville BW, Day TA: Oral cancer and precancerous lesions. CA Cancer J Clin. 2002, 52:195-215. 10.3322/canjclin.52.4.195

2. Mangold AR, Torgerson RR, Rogers RS III: Diseases of the tongue. Clin Dermatol. 2016, 34:458-469. 10.1016/j.clindermatol.2016.02.018

3. Boeing H: Alcohol and risk of cancer of the upper gastrointestinal tract: first analysis of the EPIC data . IARC Sci Publ. 2002, 156:151-154.

4. Hashibe M, Brennan P, Chuang SC, et al.: Interaction between tobacco and alcohol use and the risk of head and neck cancer: pooled analysis in the International Head and Neck Cancer Epidemiology Consortium. Cancer Epidemiol Biomarkers Prev. 2009, 18:541-550. 10.1158/1055-9965.epi-08-0347

5. Rhodus NL, Kerr AR, Patel K: Oral cancer: leukoplakia, premalignancy, and squamous cell carcinoma . Dent Clin North Am. 2014, 58:315-340. 10.1016/j.cden.2013.12.004

6. Neville B, Damm DD, Allen C, Chi A: Oral and Maxillofacial Pathology. Elsevier Health Sciences, 2015.

7. Scully C: RULE for cancer diagnosis. Br Dent J. 2013, 215:265-266.

8. Sathiasekar AC, Mathew DG, Lal MJ, Prakash AA, Kumar KG: Oral field cancerization and its clinical implications in the management in potentially malignant disorders. J Pharm Bioallied Sci. 2017, 9:23-25. 10.4103/jpbs.JPBS_109_17 


\section{Cureus}

9. Mohan M, Jagannathan N: Oral field cancerization: an update on current concepts . Oncol Rev. 2014, 8:244. 10.4081/oncol.2014.244 